\title{
Improved in vivo photoacoustic microscopy based on a virtual-detector concept
}

\author{
Meng-Lin Li, Hao F. Zhang, and Konstantin Maslov \\ Optical Imaging Laboratory, Department of Biomedical Engineering, Texas A\&M University, 3120 TAMU, \\ College Station, Texas 77843-3120 \\ George Stoica \\ Department of Veterinary Pathobiology, Texas A\&M University, 5547 TAMU, College Station, Texas 77843-5547
}

Lihong V. Wang

Optical Imaging Laboratory, Department of Biomedical Engineering, Texas A\&M University, 3120 TAMU,

College Station, Texas 77843-3120

Received July 18, 2005; revised October 18, 2005; accepted November 4, 2005; posted November 10, 2005 (Doc. ID 63562 )

Recently an in vivo high-resolution backward-mode photoacoustic microscope was developed that shows potential for applications in dermatology and related cancer research. However, the limited depth of focus of the large-numerical-aperture (NA) ultrasonic lens employed in this system causes the image quality to deteriorate significantly in the out-of-focus region. To solve this problem, we devised and explored, for the first time to our knowledge, a virtual-detector-based synthetic-aperture focusing technique, combined with coherence weighting, for photoacoustic microscopy with such a large-NA transducer. Images of phantoms show that the proposed technique improves the $-6 \mathrm{~dB}$ lateral resolution from 49-379 to $46-53 \mu \mathrm{m}$ and increases the signal-to-noise ratio by up to $29 \mathrm{~dB}$, depending on the distance from the ultrasonic focal point. In vivo experiments show that the technique also provides a clearer representation of the vascular distribution in the rat's scalp. (C) 2006 Optical Society of America

OCIS codes: $170.3880,170.5120,180.5810$.

Photoacoustic imaging, which takes advantage of high optical contrast and low acoustical scattering, has been applied to measuring the optical properties of biological tissues noninvasively. It is a promising tool for vasculature structural imaging, ${ }^{1-3}$ breast tumor detection, ${ }^{4}$ epidermal melanin measurement, ${ }^{5,6}$ and oxygenation monitoring in blood vessels. ${ }^{7}$ Recently an in vivo backward-mode confocal photoacoustic microscope (PAM) with dark-field illumination was invented by Maslov et al. to image blood vessels in the skin. ${ }^{8}$ This system provides high lateral resolution ( $45 \mu \mathrm{m}$ at the focal point) and high axial resolution $(\sim 15 \mu \mathrm{m})$, and it is capable of imaging optical absorption contrast as deeply as $3 \mathrm{~mm}$ in biological tissue. This PAM system shows potential for applications in dermatology and related cancer research. To achieve high image resolution and high sensitivity, this system employs a high-frequency, wideband, large-numerical-aperture (NA) spherically focused ultrasonic transducer that is coaxial and confocal with the optical illumination. The trade-off in using such a large-NA ultrasonic lens, however, is limited depth of focus, which causes the image quality to deteriorate significantly in the out-of-focus region. Liao et al. presented the idea of using a synthetic-aperture focusing technique (SAFT) to improve the resolution and the signal-to-noise ratio (SNR) in photoacoustic imaging, in which a point detector (e.g., a needle hydrophone) is required ${ }^{9}$ Here, we investigate a virtual-point-detector-based SAFT combined with coherence weighting to extend the depth of focus. The virtual-point-detector concept enables the SAFT to be applied to a large-NA transducer.
In this virtual-detector SAFT, we consider the focal point of the transducer to be a virtual point detector, as illustrated in Fig. 1(a). When biological tissues absorb pulsed laser energy, photoacoustic (PA) waves that are generated within a certain solid angle are assumed to be detected by the virtual detector. If a linear scan is performed, the superposition of the PA radiation patterns from the virtual detectors at adjacent positions facilitates synthetic-aperture focusing in the overlapped region above and below the virtual detectors [e.g., the two filled circles in the overlapped $\mathrm{PA}$ radiation patterns of the two transducer positions in Fig. 1(a)].

The virtual-detector based SAFT consists of applying appropriate delays relative to the virtual point detector to the adjacent scan lines and then summing the delayed signals $^{10}: \quad S_{\mathrm{SAFT}}(t)=\Sigma_{i=0}^{N-1} S\left(i, t-\Delta t_{i}\right)$,

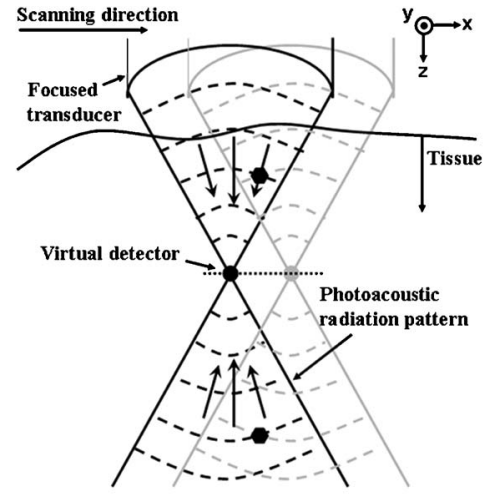

(a)

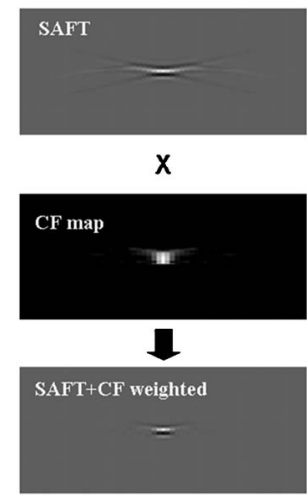

(b)
Fig. 1. (a) Schematic of the virtual-detector concept; (b) illustration of $\mathrm{CF}$ weighting. 
where $S(i, t)$ is the received signal at scan line $i$ and $\Delta t_{i}$ is the calculated acoustic propagation time from the synthetic focal point to the virtual detector at scan line $i$. $N$, the maximum number of scan lines included in the sum, is determined by the angular extent of the PA radiation pattern, which is related primarily to the parameters of the ultrasonic transducer as the distribution of light is much broader owing to the strong light scattering in biological tissue. Note that a negative time delay is used if the synthetic focal point is shallower than the focal point of the transducer. In addition to improving the lateral resolution in the out-of-focus region, the SAFT can increase the SNR as well.

In addition to this virtual-detector SAFT, a coherence factor $(\mathrm{CF})$ of the delayed signals for each SAFT imaging point can be used as a weighting factor to further improve the focusing quality. The $\mathrm{CF}$ is defined as $\mathrm{CF}(t)=\left|\sum_{i=0}^{N-1} S\left(i, t-\Delta t_{i}\right)\right|^{2} /\left[N \Sigma_{i=0}^{N-1} \mid S(i, t\right.$ $\left.\left.-\Delta t_{i}\right)\left.\right|^{2}\right]$ and is a real quantity that ranges from 0 to 1 , indicating the degree of in-phase addition among the delayed signals. ${ }^{9}$ The idea of $\mathrm{CF}$ weighting is illustrated in Fig. 1(b), where the vertical axis is the depth and the horizontal axis denotes the lateral position. The top of Fig. 1(b) shows the simulated pointspread function in the SAFT where the point is located at a depth below the focal point of the transducer. The middle of Fig. 1(b) is the corresponding CF map. The definition of the CF implies that, because of the constructive summation of the delayed signals (i.e., they are highly in phase), the $\mathrm{CF}$ at the main lobe of the point-spread function is high (close to 1), whereas the CFs at the sidelobes are low because of destructive summation. Based on this property, the sidelobes in the SAFT are suppressed after being multiplied by the corresponding CF map on a point-by-point basis; thus the beam quality is improved, as shown at the bottom of Fig. 1(b). CF weighting can also reduce the elevated sidelobes in the SAFT that result from sound velocity inhomogeneity in the tissue. ${ }^{11}$ In addition, it can suppress background noise because measurement noise is generally out of phase.

The in vivo dark-field backward-mode PAM developed by Maslov et al. was used in this study. ${ }^{8} \mathrm{~A}$ tunable dye laser (ND6000, Continuum) pumped by a Nd:YAG laser (Brilliant B, Bigsky) was employed to provide laser pulses with a FWHM of $6.5 \mathrm{~ns}$, a pulse repetition rate of $10 \mathrm{~Hz}$, and a wavelength of $584 \mathrm{~nm}$. The laser energy was delivered by a $0.6 \mathrm{~mm}$ diameter optical fiber whose output was coaxially positioned on a three-dimensional precision mechanical scanner driving an ultrasonic transducer of $50 \mathrm{MHz}$ center frequency and 70\% nominal bandwidth (V214-BCRM, Panametrics). A homemade concave lens provided the transducer with a NA of 0.44 and a focal length of $6.7 \mathrm{~mm}$. The incident energy density on the sample surface was controlled to $<1 \mathrm{~mJ} / \mathrm{cm}^{2}$. The PA signals were acquired at a $250 \mathrm{MHz}$ sampling rate and were recorded by a digital oscilloscope (TDS 5034B, Tektronics) through a broadband amplifier (ZFL-500N, Mini-Circuits). In the image formation calculation of all the following experiments, the acoustic velocity was assumed to be $1.5 \mathrm{~mm} / \mu \mathrm{s}$. In the SAFT, each scan line was first interpolated by a factor of 8 to increase the delay accuracy. Note that the PA images shown here were taken without signal averaging to minimize the data acquisition time, and the brightness of the images represents the photoacoustic pressure detected by the transducer.

The point-spread function of the PAM was evaluated by a cross-sectional view of a $6 \mu \mathrm{m}$ carbon fiber immersed in a $1 \%$ Intralipid solution (Clintec Nutrition Company, Deerfield, Ill.). This fiber was imaged at various depths, and images at different depths were montaged to one image for ease of display. The scanning step size was $20 \mu \mathrm{m}$. Note that the maximum number of scan lines for the beam synthesis was limited to 40 in this case. Figure 2 shows the original B-scan image [Fig. 2(a)] and the SAFT+CF weighted image [Fig. 2(b)]. The two images are shown on the same linear gray scale. The vertical axis is the depth from the transducer surface, and the horizontal axis is the lateral position. In Fig. 2(a), because of the limited depth of focus of the transducer, the farther the carbon fiber is away from the focal point, the wider the lateral extent, which indicates poorer lateral resolution. The $\mathrm{SAFT}+\mathrm{CF}$ weighted image exhibits improved lateral resolution. The $-6 \mathrm{~dB}$ lateral and axial resolution and the improved SNR at all the imaging depths are given in Table 1, in which the SNR is defined as the ratio of the maximal intensity at the carbon fiber position to the average noise intensity. The $\mathrm{SAFT}+\mathrm{CF}$ weighting leads to depth-independent lateral resolution, reducing the $-6 \mathrm{~dB}$ width of the imaged carbon fiber from $49-379$ to $46-53 \mu \mathrm{m}$, depending on the distance from the ultrasonic focal point, without affecting the axial resolution (estimated $-6 \mathrm{~dB}$ axial resolution, $\sim 11 \mu \mathrm{m})$. Table 1 shows that the estimated $-6 \mathrm{~dB}$ lateral resolution at depths of 5.8 and $7.6 \mathrm{~mm}$, which are farther from the focal point, is better than those at the depths of 6.1 and $7.3 \mathrm{~mm}$. This is a result of the dark-field illumination in the PAM system. Moreover, the SAFT $+\mathrm{CF}$ weighting improves the SNR by up to $29 \mathrm{~dB}$. Note that the SNR is not improved at
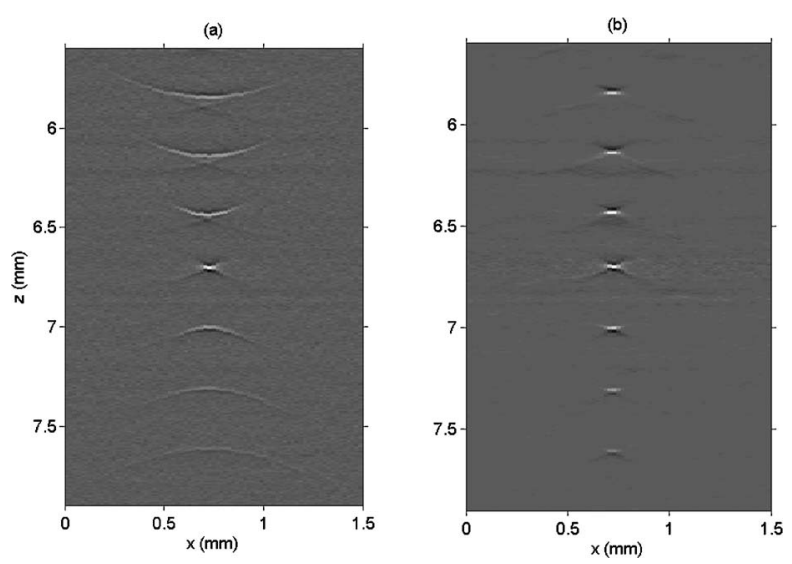

Fig. 2. B-scan images of an Intralipid phantom containing an embedded $6 \mu \mathrm{m}$ carbon fiber, which was scanned at various depths: (a) original image, (b) SAFT $+\mathrm{CF}$ weighted image. 
Table 1. Improvement of the $-6 \mathrm{~dB}$ Lateral and Axial Resolution as Well as the SNR

\begin{tabular}{llllllll}
\hline & \multicolumn{7}{c}{ Depth from the Transducer $(\mathrm{mm})$} \\
\cline { 2 - 8 } Systems and Improved SNR & 5.8 & 6.1 & 6.4 & 6.7 & 7 & 7.3 & 7.6 \\
\hline $\begin{array}{l}\text { Original system } \\
\quad(\text { lateral/axial, } \mu \mathrm{m} / \mu \mathrm{m})\end{array}$ & $259 / 11$ & $379 / 12$ & $203 / 12$ & $49 / 11$ & $153 / 12$ & $243 / 11$ & $225 / 13$ \\
$\begin{array}{l}\text { SAFT+CF weighted system } \\
\quad(\mu \mathrm{m} / \mu \mathrm{m})\end{array}$ & $49 / 11$ & $48 / 11$ & $46 / 10$ & $49 / 11$ & $51 / 11$ & $53 / 13$ & $51 / 11$ \\
\begin{tabular}{l} 
Improved SNR (dB0) \\
\hline
\end{tabular} & 29 & 21 & 19 & 0 & 16 & 25 & 24 \\
\hline
\end{tabular}
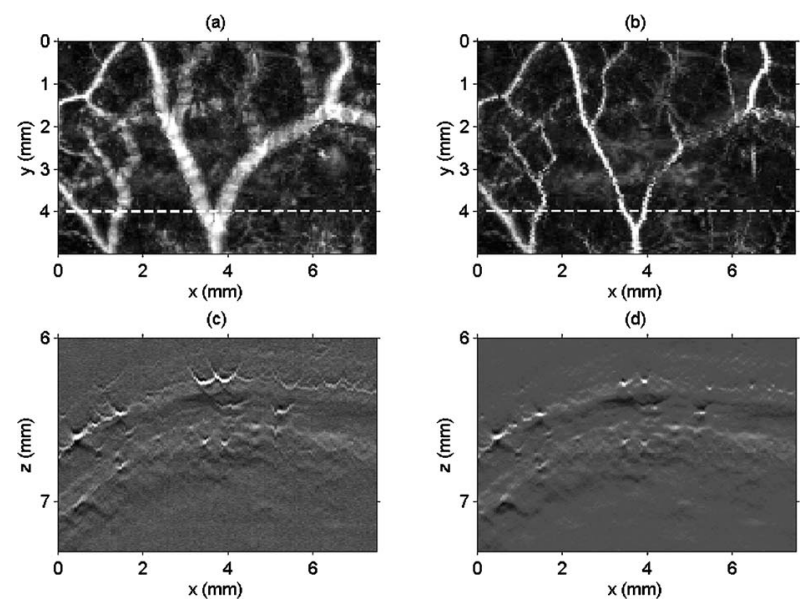

Fig. 3. (a), (b) In vivo projected C-scan images of the rat's scalp; (c), (d) in vivo B-scan images that correspond to the scanning positions indicated by the white dashed lines in (a) and (b), respectively. (a), (c) original; (b), (d) SAFT+CF weighted.

the focal point because no actual SAFT summing is performed.

We also conducted in vivo experiments with rats to further evaluate the efficacy of the proposed technique. Scalps of Sprague Dawley rats $(\sim 100 \mathrm{~g}$; Charles River Breeding Laboratories) were imaged. The laboratory animal protocol for this research was approved by the University Laboratory Animal Care Committee of Texas A\&M University. To enlarge the imaging area and reduce the scanning time, we used a step size of $50 \mu \mathrm{m}$. The maximum number of scan lines for beam synthesis was limited to 10 to reduce motion artifacts. A two-dimensional scan was performed in this case. Figures 3(a) (original) and 3(b) (SAFT $+\mathrm{CF}$ weighted) are in vivo projected $\mathrm{C}$-scan images of the rat's scalp showing the vascular network, in which the maximum of each scan line along the depth direction is plotted versus the twodimensional transducer position. Figures 3(c) and 3 (d) show the in vivo original and the SAFT $+\mathrm{CF}$ weighted B-scan images that correspond to the scanning positions indicated by the white dashed lines in Figs. 3(a) and 3(b), respectively. Note that SAFT $+\mathrm{CF}$ weighting was performed for each $\mathrm{B}$ scan along the $x$ direction; hence only the lateral resolution in the $x$ direction can be improved. Figure 3(b) provides a clearer vascular distribution as a result of the improved lateral resolution and SNR, as shown in Fig. $3(d)$, which exhibits superior definition of the vessel positions compared with the original image in Fig. 3(c) over the entire depth of the imaging field.

In summary, the virtual-detector focusing technique significantly improves the degraded lateral resolution in the out-of-focus region of a PAM system that employs a large-NA ultrasonic transducer. The phantom and in vivo experimental results demonstrate the efficacy of this technique: Depthindependent lateral resolution is achieved, and the SNR is also improved. The extended depth of focus for the PAM system enables a three-dimensional reconstruction of the vascular network to be made for the study of tumor angiogenesis. In addition, if a higher-pulse-rate laser is used to increase the scanning speed and thus to reduce motion artifacts, a two-dimensional SAFT can be used to produce even better image quality.

This project was sponsored by National Institutes of Health grants R01 EB000712 and R01 NS46214. L. V. Wang's e-mail address is lwang@oilab.tamu.edu.

\section{References}

1. X. Wang, Y. Pang, G. Ku, X. Xie, G. Stoica, and L. V. Wang, Nat. Biotechnol. 21, 803 (2003).

2. G. Ku, X. Wang, X. Xie, G. Stoica, and L. V. Wang, Appl. Opt. 41, 770 (2005).

3. J. J. Niederhauser, M. Jaeger, R. Lemor, P. Weber, and M. Frenz, IEEE Trans. Med. Imaging 24, 436 (2005).

4. R. O. Esenaliev, A. A. Karabutov, and A. A. Oraevsky, IEEE J. Sel. Top. Quantum Electron. 5, 981 (1999).

5. E. V. Savateeva, A. A. Karabutov, B. Bell, R. Johnigan, M. Motamedi, and A. A. Oraevsky, in Proc. SPIE 3597, $55(2000)$.

6. J. A. Viator, L. O. Svaasand, G. Aguilar, B. Choi, and J. S. Nelson, in Proc. SPIE 4960, 14 (2003).

7. R. O. Esenaliev, I. V. Larina, K. V. Larin, D. J. Deyo, M. Motamedi, and D. S. Prough, Appl. Opt. 41, 4722 (2002).

8. K. Maslov, G. Stoica, and L. V. Wang, Opt. Lett. 30, 625 (2005).

9. C.-K. Liao, M.-L. Li, and P.-C. Li, Opt. Lett. 29, 2506 (2004).

10. M.-L. Li, W. J. Guan, and P.-C. Li, IEEE Trans. Ultrason. Ferroelectr. Freq. Control 51, 63 (2004).

11. P.-C. $\mathrm{Li}$ and M.-L. Li, IEEE Trans. Ultrason. Ferroelectr. Freq. Control 50, 128 (2003). 